\title{
Criminologie
}

\section{Les réincarcérations multiples : profil sexué d'un groupe de justiciables}

\author{
Shirley Roy, Danielle Laberge et Marie-Marthe Cousineau
}

Volume 25, numéro 1, 1992

Les femmes et le contrôle pénal, questions féministes

URI : https://id.erudit.org/iderudit/017317ar

DOI : https://doi.org/10.7202/017317ar

Aller au sommaire du numéro

\section{Éditeur(s)}

Les Presses de l'Université de Montréal

ISSN

0316-0041 (imprimé)

1492-1367 (numérique)

Découvrir la revue

Citer cet article

Roy, S., Laberge, D. \& Cousineau, M.-M. (1992). Les réincarcérations multiples : profil sexué d’un groupe de justiciables. Criminologie, 25(1), 101-117.

https://doi.org/10.7202/017317ar
Résumé de l'article

Women offenders had traditionally been neglected in criminological theory as well as in empirical analyses. Feminist studies have shown that such an exclusion was not only inacceptable on a political ground but that it also shed serious doubts about the validity of criminological models. Arguing on the necessity of focussed empirical analyses for a better understanding of the dynamics of sexual identity on the nature of penal interventions, the authors have proceeded to a comparative analysis of the characteristics and penal treatment of a particular group of offenders, those that have been incarcerated in Quebec's provincial jails ten times or more during a ten year period. Results stress the complexity of the différenciation process for women and men, at least for this particular group. Women having been through repeated incarcerations are far less numerous than men. But the motives for which these women were imprisoned appear to be even more trivial than those having prompted the men's incarcerations. 
LES RÉINCARCÉRATIONS MULTIPLES : PROFIL SEXUÉ D'UN GROUPE DE JUSTICIABLES ${ }^{1}$

Shirley ROY ${ }^{2}$, Danielle LABERGE ${ }^{3}$, Marie-Marthe COUSINEAU ${ }^{4}$

Women offenders had traditionally been neglected in criminological theory as well as in empirical analyses. Feminist studies have shown that such an exclusion was not only inacceptable on a political ground but that it also shed serious doubts about the validity of criminological models. Arguing on the necessity of focussed empirical analyses for a better understanding of the dynamics of sexual identity on the nature of penal interventions, the authors have proceeded to a comparative analysis of the characteristics and penal treatment of a particular group of offenders, those that have been incarcerated in Quebec's provincial jails ten times or more during a ten year period. Results stress the complexity of the differenciation process for women and men, at least for this particular group. Women having been through repeated incarcerations are far less numerous than men. But the motives for which these women were imprisoned appear to be even more trivial than those having prompted the men's incarcerations.

Depuis une vingtaine d'années, les études empiriques et les efforts de théorisation portant sur les femmes se sont considérablement multipliés en criminologie. Ces travaux examinent une diversité importante de thèmes: les femmes criminalisées, la victimisation des femmes, le rôle des femmes agentes de contrôle social, le traitement passé et actuel des femmes dans les théories criminologiques, le traitement des femmes par les agences pénales.

1. Ces résultats s'inscrivent dans le cadre d'une subvention de recherche subventionné par le FCAR, volet « actions spontanées ». Les auteures désirent remercier Julcs Duchastel pour ses commentaires qui nous ont été fort utiles.

2. Boursière post-doctorale au Laboratoire de changement social, Universite Paris VII ; membre du Groupe de recherche et d'analyse sur les politiques et les pratiques pénales (GRAPPP), C.P. 8888, succ. «A ", Montréal (Québec) H3C 3P8.

3. Professeure, département de sociologie de I'UQAM ; membre du Groupe de recherche et d'analyse sur les politiques et les pratiques penales (GRAPPP), C.P. 8888, succ. "A ", Montréal (Québec) H3C 3P8.

4. Chercheure, département de sociologie de l'UQAM ; membre du Groupe de recherche et d'analyse sur les politiques et les pratiques pénales (GRAPPP), C.P. 8888, succ. "A ", Montréal (Québec) H3C 3P8. 
L'émergence et le développement, en criminologie, des études portant sur les femmes est surtout le fait des chercheures adoptant une perspective féministe. Une telle perspective demeure encore le fondement de la majorité des études récentes; néanmoins, elle n'est pas la seule. En effet, les études criminologiques portant sur les femmes - à un titre ou à un autre - ne peuvent plus être traitées comme un domaine de recherche acceptable mais secondaire, une sorte de champ de spécialité un peu «pointu». Le développement de modèles théoriques, qu'ils portent sur la genèse du comportement délinquant ou sur le fonctionnement des agences pénales, ne peut ignorer les caractéristiques particulières d'une partie des justiciables qui se distinguent par leur sexe. Ceci constitue, selon nous, un revirement paradigmatique important que les analystes, quelle que soit la perspective théorique adoptée, ne pourront plus ignorer.

Or, si ces questions apparaissent maintenant incontournables, un important travail d'analyse reste à accomplir. Le défi est d'ailleurs de taille: il suppose la mise au point d'une méthodologie qui tienne compte des problèmes que posent des analyses comparatives entre des groupes de tailles disproportionnées, ou encore des analyses centrées strictement sur un groupe, ici les femmes, avec leurs caractéristiques propres, de même que le découpage de plans ou de domaines d'analyse qui permettent la réintroduction des femmes comme sujets particuliers de contrôle social, le développement d'un important corpus d'études empiriques...

L'élaboration de modèles théoriques portant globalement sur les formes sexuées du contrôle social est essentielle pour une meilleure compréhension des modes de reproduction sociale. Il nous semble pourtant que, jusqu'à maintenant et pour des raisons fort compréhensibles, on ait négligé un travail d'analyse plus empirique et surtout plus précis quant à ces objets. En effet, et particulièrement lorsqu'il s'agit du contrôle social de nature pénale, la dynamique des rapports de sexe n'est pas évidente et ne pourra être mise au jour si l'analyse traite toujours le système pénal comme un tout cohérent dont chaque partie serait interchangeable.

C'est donc dans le cadre d'une analyse circonscrite que se situe notre contribution. Le travail présenté ici porte sur une analyse comparative femmes/hommes des profils d'incarcération d'une sous-catégorie de justiciables, ceux et celles ayant fait l'objet d'incarcérations multiples sur une période de quelques années. Dans un premier temps, nous présenterons brièvement les paramètres théoriques et méthodologiques 
présidant à notre analyse. Dans un deuxième temps, nous en commenterons les résultats. En dernier lieu, nous discuterons des avenues qu'ouvrent de telles analyses.

\section{PERSPECTIVE THÉORIQUE ET CHOIX EMPIRIQUES}

Notre hypothèse de départ est l'existence d'un traitement pénal différencié entre les hommes et les femmes. Cette différenciation n'est pas pensée ici en termes de traitement préférentiel ou discriminatoire d'un groupe ou de l'autre, perspective que l'on retrouve souvent dans les écrits des dix dernières années ${ }^{5}$. La reconnaissance de la différenciation et de ses manifestations particulières relève, dans un premier temps, de l'ordre du constat: les femmes et les hommes n'ont pas le même profil de criminalité ni le même profil de prise en charge pénale. Ces différences peuvent s'expliquer de diverses façons. Disons rapidement ici que la production particulière des rapports de genre, liés au sexe des personnes, est antérieure à la criminalisation des justiciables ${ }^{6}$. En d'autres termes, les comportements délinquants, tout comme les interventions pénales, permettent de saisir les représentations et les rapports sociaux qui définissent «ce qu'est » et, par conséquent, «comment devrait se comporter » une femme ou un homme.

Nous avons traité le système pénal comme un tout, ce qui se justifie à plusieurs égards. Pourtant, à la fois pour des raisons thériques et à cause des contraintes réelles de la recherche empirique, on doit procéder à des distinctions, entre autres, entre les différentes agences qui composent le système pénal. Théoriquement, rien ne permet de croire que les mécanismes de différenciation sur la base du sexe soient les mêmes d'une agence à l'autre. Au contraire, les études sont nombreuses qui illustrent les variations importantes dans les modes d'intervention et de fonctionnement de la police, du tribunal et du système correctionnel, par exemple. Ces variations sont susceptibles de se traduire dans le traitement particulier que l'on réserve à des groupes de justiciables, ici les femmes et les hommes. Sur le plan empirique, ces distinctions sont essentielles si l'on souhaite procéder à des études minutieuses contribuant à la constitution d'un corpus de recherche qui demeure bien

5. Voir Parent (1986) pour un examen critique de l'idée de traitement préférentiel des femmes ; voir Laberge (1990) pour une analyse des problèmes liés au débat préférentiel-discriminatoire.

6. Cette question théorique extrêmement importante déborde largement le cadre du présent article. À ce sujet, on pourra consulter entre autres Mathicu (1989). 
restreint. Elles seules sont susceptibles de mettre au jour les modalités particulières de constitution de l'identité sexuelle des justiciables.

Nous avons opté ici pour l'étude quantitative ${ }^{7}$ d'une partie de la population carcérale. L'examen de ce groupe particulier de justiciables permet d'identifier les comportements ou les situations qui sont gérées en utilisant une forme extrêmement sévère de sanction, la privation de liberté. Les prisons du Québec admettent, comme on le sait, les personnes ayant reçu une sentence d'incarcération inférieure à deux ans ainsi que celles retenues en détention provisoire. Dans le cadre de notre étude, nous avons eu accès aux données du ministère de la Sécurité publique du Québec concernant l'ensemble des personnes incarcérées au cours de la période 1977-1985. Notre analyse porte donc sur la population des personnes incarcérées et non pas sur un échantillon de celles-ci.

Tout d'abord, rappelons que le phénomène de l'incarcération n'est pas banal : au cours de la période retenue, on a enregistré, dans les prisons québécoises, 289693 admissions touchant plus de 130000 personnes différentes, dont $119276(88 \%)$ étaient des hommes et 10751 ( $12 \%$ ) des femmes. Parmi cette population, certains justiciables n'ont connu l'emprisonnement qu'une seule fois (ceci ne présumant en rien de la fréquence de leurs contacts avec la police ou les tribunaux) ${ }^{8}$. d'autres ont été incarcérés plus ou moins fréquemment pour des périodes plus ou moins longues. C'est à ce dernier groupe que nous nous sommes intéressées. Il nous faut maintenant clarifier la perspective adoptée dans la constitution du sous-groupe particulier que nous étudions.

La notion de récidiviste a été et est encore utilisée pour désigner les justiciables qui ont connu plus d'un contact avec le système pénal; elle s'applique tout particulièrement aux personnes faisant l'objet de plus

7. Il aurait aussi êté intéressant de procéder à une analyse qualitative. Nous avons opté en premier lieu pour l'étude quantitative, puisque nous cherchions avant tout à identifier des effets du système tels qu'ils peuvent se lire dans le traitement différencié et particulier de fractions spécifiques des justiciables.

8. En effet, on peut sans contredit considérer que les contacts pénaux donnant lieu à une incarcération provisoire ou punitive ne représentent que la pointe de l'iceberg. Il est des prévenus qui se présenteront libres aux différentes étapes de leur procès et qui, au terme des procédures, ou bien seront disculpés, ou bien, s'ils sont reconnus coupables, se verront imposer des sentences autres que l'emprisonnement. Il reste que pour tous ceux-là, les moments mobilisés par les procédures fudiciaires sont aussi contraignants et entrainent souvent des coûts sociaux considérables (Landreville, Pires et Blankevoort, 1981 ; Imbleau, 1988). 
d'une sentence d'incarcération. Les limites et les ambiguîtés de cette notion pour désigner des individus ayant des contacts multiples avec le système pénal ont été largement discutées (Landreville, 1982). Afin de lever toute ambiguiité, nous avons adopté, à l'instar d'Imbleau (1988), comme désignation de la population réincarcérée à de multiples reprises l'acronyme RIM ${ }^{9}$.

La notion de RIM (réincarcérations multiples) étant une construction a posteriori, il fallait d'abord lui donner une matérialité et arrêter la façon de l'opérationnaliser. Le choix de la coupure a été fixé à 10 retours au cours de la période 1977-1985. Ainsi, pour être sélectionné comme sujet d'étude, un individu devait avoir cumulé au moins 10 incarcérations à un titre ou à un autre (prévenu ou détenu) dans l'une ou l'autre des prisons du Québec sur une période de neuf ans (ce qui représente une fréquence d'un peu plus d'une incarcération annuellement). Ce choix, quoique arbitraire, nous permet de nous concentrer sur les cas extrêmes, ceux chez qui devraient se retrouver les traces les plus marquées de l'effet du système pénal.

\section{UN EXAMEN EMPIRIQUE ${ }^{10}$}

Le groupe des personnes RIM représente 2 pour cent de l'ensemble de la population incarcérée entre 1977-1985, soit 2615 individus. L'écart entre la représentation des femmes et des hommes y est encore plus marqué que lorsque l'on utilise les statistiques des admissions sans distinction quant aux types de justiciables. Dans le cas des justiciables RIM, les hommes sont 32 fois plus nombreux que les femmes: les premiers représentent 97,3 pour cent de la population RIM et les secondes comptent pour 2,7 pour cent. On observe une légère différence dans l'étendue du nombre d'admissions. Alors que les femmes RIM se sont retrouvées en prison 24,7 fois en moyenne au cours de la période étudiée (de 10 à 117 admissions), la moyenne du nombre de réadmissions se situe à 20,1 fois chez les hommes (de 10 à 149 admissions). Les 71 femmes RIM totalisent, durant la période à l'étude, 1755 admissions; les 2544 hommes RIM sont à l'origine, pour leur part, de 51135 admissions.

9. Nous utilisons l'acronyme RIM pour désigner certains groupes de justificiables afin d'éviter des lourdeurs stylistiques. Il ne s'agit nullement pour nous de réduire l'expérience pénale, voire personnelle, de ces justiciables a cette seule réalité.

10. Une partie de ce matériel a fait l'objet d'une analyse dans la thèse de S. Roy (1990). 
En premier lieu, nous désirons insister sur l'importance matérielle remarquable que prennent un très petit nombre de justiciables dans le volume total des admissions dans les prisons du Québec. En effet, 2 pour cent des personnes sont responsables de 17,6 pour cent des admissions. De toute évidence, le seuil minimal de 10 admissions servant à définir de façon opératoire la notion de réincarcérations multiples (RIM) contribue à cet important volume. De fait, la moyenne des admissions pour l'ensemble des justiciables de notre sous-groupe dépasse largement ce seuil.

C'est donc dire qu'il s'agit d'un sous-groupe ayant nécessairement des caractéristiques particulières sur le plan de leur histoire pénale et sociale. Dans le cas de ces justiciables, il est en effet impossible de penser leur trame de vie en termes d'insertion sociale "normale ", la simple gestion de tout ce qui entoure une cause - comparution, remise, rencontres avec l'avocat de la défense, incarcération - constitue, pour beaucoup, une occupation a temps plein. C'est aussi un portrait fort différent des "récidivistes classiques" que l'on retrouve parmi ce groupe : comme nous le verrons plus loin, ces justiciables se caractérisent par la banalité de leurs délits et des sentences qu'ils encourent.

Par ailleurs, le phénomène RIM doit être envisagé sur la base des rapports de sexe. Les femmes contribuent de façon très restreinte à l'alimentation du système pénal; cette contribution va d'ailleurs en diminuant au fil des étapes du processus pénal (Hamelin, 1985). Ce phénomène d'attrition se retrouve aussi, et ce de façon encore plus marquée, dans l'évaluation de l'intensité du contact avec le pénal. En effet, la notion de RIM, telle que nous l'avons définie, constitue un indicateur de l'intensité des contacts qu'un individu donné a avec le système pénal, contacts qui se traduisent par une mesure d'incarcération. Dans le cas des femmes, on constate, d'une part, qu'elles sont moins fréquemment en contact avec le système pénal et, d'autre part, que parmi celles qui sont en contact, une proportion plus faible a un contact intense $(0,7 \%$ des femmes et $2,3 \%$ des hommes). Ceci ne présume en rien de la sévérité plus ou moins grande dont feraient l'objet les femmes justiciables.

\subsection{Un portrait socio-démographique}

Les caractéristiques socio-démographiques des justiciables RIM à leur première admission en prison montrent de très légères différences entre les hommes et les femmes et correspondent, dans l'ensemble, au 
portrait de la majorité des justiciables qui en sont à leur premier séjour en prison. Ils sont relativement jeunes, peu scolarisés et célibataires.

Âge et scolarité à la première admission pour les femmes et les hommes

\begin{tabular}{lllcc}
\hline & Áge à la l'e admission & \multicolumn{2}{c}{$\begin{array}{c}\text { Scolarité complétée à la } \\
\text { re admission }\end{array}$} \\
& Femmes & Hommes & Femmes & Hommes \\
\hline $\begin{array}{l}\text { Moyenne } \\
\text { Médiane }\end{array}$ & 25,5 & 24,9 & 7,9 & 8,2 \\
\hline
\end{tabular}

Sur le plan économique, c'est un groupe qui est fort probablement démuni $^{11}$. Toujours à la première admission, qui ne se distingue pas des autres moments d'admission sur ce plan, 85,7 pour cent des femmes et 63,7 pour cent des hommes déclarent n'avoir aucun emploi. Parmi ceux et celles qui travaillent, on retrouve les femmes essentiellement (près des deux tiers) dans des occupations que l'on se voit contraint de classer sous la rubrique « autres », c'est-à-dire des occupations non définies et non qualifiées. Les hommes, pour leur part, occupent quatre secteurs d'emplois : artisanat/ouvrier $(31,4 \%)$, manœuvre $(29,9 \%)$, autres $(14,4 \%)$ et construction $(11,5 \%)$. La chose intéressante à retenir ici, c'est que lorsque l'on compare les justiciables RIM à la population québécoise, ceux-ci se retrouvent dans une position complètement inversée dans leur rapport au travail. Alors que pour des tranches d’âge comparables les femmes de la population québécoise sont à 76,6 pour cent sur le marché du travail, seulement 15 pour cent des femmes-RIM sont dans la même situation. En ce qui concerne les hommes, bien que l'écart soit moins important, on constate que 92 pour cent des hommes québécois (des mêmes groupes d'âge) occupent un emploi contre seulement 63 pour cent des hommes incarcérés à de multiples reprises.

Ainsi, les caractéristiques des justiciables du groupe des RIM ne permettent pas de les distinguer de façon marquée des autres justiciables pour qui la première incarcération est aussi la dernière. Dans les deux cas, il s'agit de jeunes passablement démunis aussi bien sur le plan de la scolarité, donc de leur insertion potentielle sur le marché de l'emploi, que sur le plan de leur insertion véritable sur le marché du travail qui est, somme toute, peu fréquente et précaire. Notons néan-

11. Nous ne possédons pas de données concernant le revenu des justiciables. Toutefois, toute une série d'indices, nous le verrons, tendent à confirmer cette assertion. 
moins que cette précarité, qui rejette aussi bien les femmes que les hommes dans la fraction de la population la plus vulnérable, paraît encore plus marquée pour les premières que pour les seconds. Par ailleurs, du moins sur le plan des attributs socio-démographiques, rien ne permet de distinguer les justiciables, femmes ou hommes, pour qui l'incarcération se révélera une expérience unique des habitués potentiels.

\subsection{L'histoire pénale}

Pour retracer l'histoire pénale des justiciables RIM, nous avons retenu cinq variables : le tribunal ayant entendu la cause, la nature du délit, le statut à l'admission en prison, la durée de la peine et la durée de séjour. Une précaution méthodologique s'impose ici, dictée par la configuration même du phénomène à l'étude : le nombre extrêmement restreint de femmes, auquel s'ajoute le problème des informations manquantes, rend malaisée l'étude de leur groupe ainsi que la comparaison de celui-ci avec le groupe des hommes. Ce problème peut difficilement être contourné puisque nous ne travaillons pas avec un échantillon, mais bien avec l'ensemble de la population.

Généralement, les causes criminelles sont entendues devant la Cour des sessions de la paix ${ }^{12}$. Pourtant, les tribunaux de cette instance ne sont pas les seuls à avoir juridiction en cette matière. À Montréal, la cour municipale a juridiction pour entendre certaines causes de nature pénale ayant un caractère jugé moins grave. Si l'on a tendance à ignorer ce tribunal, il n'en demeure pas moins une source d'alimentation importante des prisons provinciales, leur fournissant aussi bien prévenus $(9,4 \%)$ que condamnés $(29,9 \%)^{13}$. Certains autres tribunaux ${ }^{14}$ prennent aussi des décisions en matière d'incarcération. Le tableau suivant identifie le tribunal à l'origine de l'admission pour chacun des trois moments retenus pour les fins de la comparaison, et ce pour les femmes et les hommes.

12. Maintenant désignée comme la Chambre criminelle et pénale de la Cour du Québec.

13. Pour l'ensemble de la province, pour la totalité des admissions entre 1979 et 1985. Voir Cousineau, Laberge et Théorêt (1986).

14. Principalement le Tribunal de la jeunesse, mais aussi, dans une moindre mesure, la Cour provinciale, le Cour supérieure et la Cour d'appel. 


\begin{tabular}{lcccccc}
\hline & \multicolumn{2}{c}{$\begin{array}{c}\text { Première } \\
\text { admission }\end{array}$} & \multicolumn{2}{c}{$\begin{array}{c}\text { Cinquième } \\
\text { admission }\end{array}$} & \multicolumn{2}{c}{$\begin{array}{c}\text { Dixieme } \\
\text { admission }\end{array}$} \\
& $F$ & $H$ & $F$ & $H$ & $F$ & $H$ \\
\hline Cour municipale & $35,6 \%$ & $21,7 \%$ & $38,7 \%$ & $21,6 \%$ & $45,0 \%$ & $18,7 \%$ \\
$\mathrm{~N}$ & $(21)$ & & $(24)$ & & $(27)$ & \\
Cour des sessions & $57,6 \%$ & $70,5 \%$ & $59,6 \%$ & $70,1 \%$ & $55,0 \%$ & $73,4 \%$ \\
$\mathrm{~N}$ & $(43)$ & & $(37)$ & & $(33)$ & \\
Autres tribunaux & $6,8 \%$ & $7,7 \%$ & $1,6 \%$ & $8,2 \%$ & $0,0 \%$ & $7,9 \%$ \\
$\mathrm{~N}$ & $(4)$ & & $(1)$ & & $(0)$ & \\
\hline Total & $100,0 \%$ & $100,0 \%$ & $100,0 \%$ & $100,0 \%$ & $100,0 \%$ & $100,0 \%$ \\
$\mathrm{~N}$ & $(59)$ & $(2288)$ & $(62)$ & $(2268)$ & $(60)$ & $(2260)$ \\
\hline
\end{tabular}

Il ressort de ce tableau que la cour municipale occupe une place beaucoup plus grande dans la gestion des causes pénales lorsque des femmes sont impliquées. En effet, pour les trois moments d'admission étudiés, la cour municipale a été le tribunal décisionnel dans plus du tiers de l'ensemble des causes impliquant des femmes ${ }^{15}$, alors que ceci est vrai pour les hommes dans à peine le cinquième des cas. Les autres tribunaux sont présents, à toutes fins utiles, exclusivement lorsque la cause implique un homme. Il est impossible, du moins dans le cadre de notre étude, de trancher entre diverses interprétations. Le constat est néanmoins important. La cour municipale traite des affaires banales. La décision d'orienter vers l'un ou l'autre tribunal revient généralement aux policiers ${ }^{16}$ suivant des règles prévues à cet effet. Le traitement des cas y est plus expéditif, le rôle étant particulièrement surchargé et les ressources très rares. Pourtant, la décision d'orienter vers le tribunal municipal ne constitue pas une source d'immunité quant à l'emprisonnement si l'on considère que le justiciable peut tout de même se retrouver incarcéré à un titre ou à un autre (détention provisoire ou punitive) suite à une décision de ce tribunal.

La nature des accusions, présentée au tableau suivant, pourrait apparaître comme une source d'explication. La présentation des données reprend la comparaison femmes/hommes en trois temps du tableau précédent. Ce tableau permet plusieurs types de comparaison, répondant ainsi à des questions différentes. Dans le cadre de l'analyse présentée ici, deux questions doivent être examinées : est-ce que les femmes et les hommes présentent, à chacun des moments retenus, un profil similaire

15. Les fluctuations entre ces trois périodes sont statistiquement non significatives.

16. Il arrive, bien que plus rarement, que le procureur de la poursuite choisisse de transférer vers un autre niveau de juridiction un dossier qui lui est originellement transmis. 
ou non d'accusation? est-ce que les transformations - si transformations il y a - des profils d'accusation semblent s'orienter dans une direction similaire ou non ? C'est ici le traitement des données, en termes de "carrières carcérales" des RIM, femmes et hommes, qui s'amorce.

Distribution des infractions chez la population des RIM à leurs premiere, cinquieme et dixième entrées selon le sexe des justiciables

\begin{tabular}{lcccccc}
\hline & \multicolumn{2}{c}{$\begin{array}{c}\text { Première } \\
\text { admission }\end{array}$} & \multicolumn{2}{c}{$\begin{array}{c}\text { Cinquieme } \\
\text { admission }\end{array}$} & \multicolumn{2}{c}{$\begin{array}{c}\text { Dixieme } \\
\text { admission }\end{array}$} \\
& $F$ & $H$ & $F$ & $H$ & $F$ & $H$ \\
\hline Contre la personne & $6,2 \%$ & $11,9 \%$ & $11,1 \%$ & $6,6 \%$ & $7,4 \%$ & $7,5 \%$ \\
N & $(2)$ & & $(5)$ & & $(4)$ & \\
Contre les biens & $37,5 \%$ & $40,8 \%$ & $35,6 \%$ & $32 \%$ & $44,4 \%$ & $31,1 \%$ \\
N & $(12)$ & & $(16)$ & & $(24)$ & \\
Autres Code criminel & $31,2 \%$ & $9,4 \%$ & $24,4 \%$ & $13,6 \%$ & $16,7 \%$ & $15,6 \%$ \\
N & $(10)$ & & $(11)$ & & $(9)$ & \\
Reglements & $6,2 \%$ & $5,7 \%$ & $13,3 \%$ & $8,6 \%$ & $20,3 \%$ & $8,4 \%$ \\
municipaux & $(2)$ & & $(6)$ & & $(11)$ & \\
N & - & $0,7 \%$ & - & $0,7 \%$ & $1,8 \%$ & $1,1 \%$ \\
Delits administratifs & $(0)$ & & $(0)$ & & $(1)$ & \\
N & $3,1 \%$ & $3,2 \%$ & $6,7 \%$ & $3 \%$ & - & $4 \%$ \\
Drogues & $(1)$ & & $(3)$ & & $(0)$ & \\
N & $15,6 \%$ & $28,2 \%$ & $8,9 \%$ & $35,4 \%$ & $9,3 \%$ & $32,2 \%$ \\
Circulation routière & $(5)$ & & $(4)$ & & $(5)$ & \\
$\mathbf{N}$ & $100 \%$ & $100 \%$ & $100 \%$ & $100 \%$ & $100 \%$ & $100 \%$ \\
Total & $(32)$ & $(1189)$ & $(45)$ & $(1344)$ & $(54)$ & $(1506)$ \\
\hline
\end{tabular}

Les «crimes contre les biens » apparaissent comme la catégorie stable, celle dont l'importance se maintient à chacune des admissions aussi bien pour les femmes que pour les hommes. Il n'y a rien de particulièrement surprenant dans ces résultats, puisqu'il s'agit de la catégorie d'infractions qui mobilisent le plus le système pénal. Au-delà de ce constat général, des différences s'imposent. Ainsi, l'importance de ce type de délits (comprenant des vols simples pour l'essentiel) tend à s'accroître pour les femmes ${ }^{17}$ entre la première et la dixième entrée.

17. Compte tenu du très petit nombre de cas et de la taille du tableau, il est impossible de calculer un chi carré pour évaluer statistiquement les différences observées. 
Les autres infractions au Code crimine $^{18}$ occupent une place non négligeable quoique décroissante dans le tableau de la criminalité féminine. La décroissance de cette catégorie d'infraction s'accompagne d'un accroissement important des infractions aux règlements municipaux. Cette dernière catégorie de délits concerne exclusivement l'ordre public, c'est-à-dire les comportements considérés comme inappropriés dans des lieux publics ${ }^{19}$.

Quant aux hommes, la coupe temporelie de leur profil de délit ainsi que l'évolution de ce dernier se présente de façon différente. Ainsi, l'importance de la catégorie des crimes contre les biens décroît après la première admission, tout en constituant un peu moins du tiers des délits pour lesquels ces derniers sont jugés. Dans leur cas, les infractions à la circulation routière sont une source d'emprisonnement tout aussi marquée que la catégorie précédente, prenant une importance largement supérieure à ce qu'elles représentent pour les femmes.

L'augmentation des autres infractions au Code criminel s'explique principalement par l'augmentation de ce que nous conviendrons de nommer des "délits de système" qui représentent les deux tiers de cette catégorie. Il s'agit ici d'infractions qui découlent d'un contact antérieur avec le système pénal : absence illégale, désobéissance à la cour, bris de probation... Dans de tels cas, on entrevoit la complexité de la dynamique qui produit des réincarcérations multiples.

L'accusation ayant entraîné l'incarcération du justiciable constitue un angle privilégié pour saisir le profil et l'évolution des contacts avec le système pénal. Le statut du justiciable lors de son admission permet un autre type de regard sur le phénomène et renvoie au sens particulier que prend l'incarcération pour chacune des admissions.

Sur le plan légal, on distingue : les personnes condamnées et pour qui l'emprisonnement constitue la peine ou une portion de la peine, généralement désignées comme détenu-e-s; les personnes emprisonnées en cours de procédure judiciaire et qui n'ont pas encore été condamnées ou sentencées, généralement désignées comme prévenues. Il s'agit d'une distinction fort importante, puisque l'emprisonnement n'a pas la même signification dans l'un et l'autre cas. Sur le plan administratif, la situation est plus complexe et nous nous bomerons à commenter les trois combinaisons possibles de statuts. Ces combinai-

18. Mours, paris, prostitution, possession d'armes à feu, complot, complicité, inconduite, absence illégale, désobêissance à la Cour.

19. Désordre, nưsance, ivresse, flânerie, tapage, vagabondage. 
sons proviennent de l'agencement entre le statut du justiciable à son admission en prison et son statut à la libération. On retrouve ainsi : 1) les prévenus-prévenus $(\mathbf{P} / \mathbf{P})$, c'est-à-dire les justiciables qui sont admis comme prévenus et qui sont libérés avant que les procédures ne soient terminées ou sans avoir été condamnés ou après avoir été condamnés à une sentence autre que l'emprisonnement ; 2) les prévenus-détenus $(\mathbf{P} / \mathrm{D})$, soit les justiciables qui sont admis comme prévenus et dont le statut passe à celui de détenus pendant qu'ils sont incarcérés ; 3) les détenus-détenus ( $D / D)$, soit les justiciables qui sont admis suite à une condamnation et qui, bien entendu, sont libérés après avoir purgé une partie ou la totalité de leur peine.

Trajectoire carcérale des hommes et des femmes RIM à leurs première, cinquième et dixieme admissions

\begin{tabular}{lccccccc}
\hline & \multicolumn{2}{c}{ Premiere admission } & \multicolumn{2}{c}{ Cinquième admission } & \multicolumn{2}{c}{ Dixième admission } \\
& $F$ & $H$ & $F$ & $H$ & $F$ & $H$ \\
\hline P/P & $59,1 \%$ & $47,4 \%$ & $30 \%$ & $41,2 \%$ & $38,6 \%$ & $46,1 \%$ \\
P/D & $5,6 \%$ & $12,4 \%$ & $25,7 \%$ & $17,3 \%$ & $17,1 \%$ & $15,7 \%$ \\
D/D & $35,2 \%$ & $40,1 \%$ & $44,3 \%$ & $41,5 \%$ & $44,3 \%$ & $38,2 \%$ \\
TOTAL & $100,0 \%$ & $100,0 \%$ & $100,0 \%$ & $100,0 \%$ & $100,0 \%$ & $100,0 \%$ \\
N & $(70)$ & $(2544)$ & $(70)$ & $(2544)$ & $(70)$ & $(2544)$ \\
\hline
\end{tabular}

À la lecture du tableau, une première constatation s'impose, aussi bien pour les femmes que pour les hommes: l'usage de la détention provisoire est important. Il est, dans chacun des cas (femmes et hommes à chacun des moments), au moins aussi fréquent que l'usage de la détention à des fins punitives. Compte tenu des provisions légales, l'usage de la détention provisoire devrait être beaucoup plus restreint ; cela apparaît particulièrement lorsque l'on considère la nature des délits que rapporte le tableau précédent.

Par ailleurs, on constate que la source la plus importante de variations dans le tableau, et ce aussi bien pour les femmes que pour les hommes, se situe dans les deux catégories de prévenu-e-s (P/P, P/D). Pour les femmes, les fluctuations sont significatives sur le plan statisti$q^{2} e^{20}$, sans que l'on puisse parler de tendances précises. La même chose peut être dite de la configuration générale de statuts à l'admission des hommes. Ici, la différence entre les hommes et les femmes réside dans la configuration différente de ces variations.

20. Chi carré $=16,6 \mathrm{dl}=2, \mathrm{n} . \mathrm{s}>0,001$. 
Deux dernières variables nous permettront de conclure ce portrait descriptif de l'expérience pénale des justiciables femmes et hommes RIM : la durée des peines imposées par le tribunal, dans le cas des personnes purgeant une sentence, et la durée de séjour en institution, qu'il s'agisse des P/D ou des D/D.

Durée des peines imposées en jours

\begin{tabular}{lcccccc} 
& \multicolumn{2}{c}{ Première admission } & \multicolumn{2}{c}{ Cinquième admission } & \multicolumn{2}{c}{ Dixième admission } \\
& $F$ & $H$ & $F$ & $H$ & \multicolumn{1}{c}{$F$} & \multicolumn{1}{c}{$H$} \\
\hline Moyenne & 55,0 & 85,3 & 49,9 & 92,8 & 197,7 & 101,2 \\
Médiane & 16,0 & 14,5 & 8,0 & 15,0 & 25,5 & 21,5 \\
\hline
\end{tabular}

La durée moyenne des peines imposées aux hommes et aux femmes est très différente, comme on peut le constater, et montre des tendances temporelles tout aussi distinctes. Si les peines imposées aux hommes s'accroissent constamment (en utilisant l'une et l'autre mesure de tendance centrale), celles des femmes ont un comportement beaucoup plus erratique. Bien entendu, la taille des groupes affecte ici considérablement la mesure, les informations concernant les hommes n'étant pas affectées par quelques données extrêmes, alors que toute situation de ce genre, chez les femmes, aura un impact sur la moyenne. Mais, même en utilisant la médiane, les peines ne présentent pas de tendance claire d'une admission à l'autre pour les femmes.

Durée des séjours en prison en jours

\begin{tabular}{lcccccc} 
& \multicolumn{2}{c}{ Première admission } & \multicolumn{2}{c}{ Cinquième admission } & \multicolumn{2}{c}{ Dixième admission } \\
& $F$ & $H$ & $F$ & $H$ & $F$ & $H$ \\
\hline Moyenne & 28 & 36,8 & 27 & 50,7 & 31,4 & 33,4 \\
Médiane & 4 & 7 & 7 & 7 & 2 & 7 \\
\hline
\end{tabular}

La lecture que l'on peut faire des informations concernant les durées de séjour n'est pas simple. Une double contingence joue ici. D'une part, on retrouve des prévenus et des détenus dont la durée de séjour est affectée par des facteurs très différents. D'autre part, le tribunal impose une peine à titre de sentence, mais la gestion matérielle de celle-ci revient à l'administration carcérale. Ces deux éléments sont affectés de façon différente par la conjoncture particulière à une période donnée. Ainsi, l'engorgement des tribunaux est susceptible d'affecter à la 
hausse la durée de séjour des personnes prévenues; dans ce cas, les autorités carcérales n'ont pas la latitude de libérer le justiciable. Par ailleurs, la surpopulation carcérale affectera à la baisse la durée de séjour des personnes sentencées, puisque les institutions ne peuvent garder toutes les personnes qui sont envoyées par le tribunal pour la totalité de leur peine. La moyenne que l'on obtient est le résultat composite de ces divers éléments dont l'importance relative est impossible à déterminer.

\section{QU'EST-CE QUE LA DIFFÉRENCE ?}

Comme on aura pu le constater, la comparaison entre les femmes et les hommes justiciables est un exercice périlleux qui soulève autant de questions qu'il apporte de réponses. Les résultats présentés ici ont permis d'ébaucher un portrait des justiciables femmes et hommes ayant connu des réincarcérations multiples. Cette ébauche servait de base à une exploration de la construction différenciée des profils pénaux sur la base du sexe des justiciables. Quelles conclusions tirer de ces analyses? Les quelques caractéristiques socio-démographiques pour lesquelles nous disposions d'informations laissent entrevoir peu de différences entre femmes et hommes, justiciables dont le portrait se confond avec celui de l'ensemble des populations incarcérées au Québec. L'examen des variables ayant un caractère directement pénal nous permet, quant à lui, quelques constatations importantes. Premièrement, les justiciables RIM se démarquent clairement, de tous les points de vue, de l'image traditionnelle du récidiviste: délits de peu d'importance, banalisation des délits à travers le temps, durée d'incarcération relativement courte. Ajoutons à cela le caractère quasi indifférencié du statut à l'admission des justiciables, en d'autres termes, l'étonnante présence des admissions à titre de personnes prévenues dans la trajectoire carcérale du groupe à l'étude.

Sur le plan de la comparaison entre les sexes, les pistes se brouillent. D'une variable à l'autre, on décèle certaines différences. Ainsi, le profil des délits est clairement différent pour les hommes et pour les femmes ; ceci est vrai de façon générale, et ce, quelle que soit l'étape du processus judiciaire où l'on procède à la comparaison. À notre avis, ces résultats constituent, par la négative, une base pour rejeter la thèse du traitement préférentiel des femmes. En effet, si les femmes jouissaient d'un traitement plus favorable, de façon générale, que celui réservé aux hommes, celles que l'on retrouve en prison sur une base systématique 
(les RIM) devraient avoir un profil de délits plus sérieux que celui des hommes dans une situation similaire. En d'autres termes, pour des mesures pénales équivalentes, les femmes devraient présenter un profil de délinquance plus sérieux. Or, les femmes-RIM ne se distinguent pas par la gravité des délits pour lesquels elles sont incriminées.

A certains égards, le traitement pénal que subissent les femmes RIM semble plus sévère que celui des hommes. Nous pensons particulièrement ici aux infractions aux règlements municipaux et aux incarcérations générées par la cour municipale qui occupent une place importante dans le profil des femmes RIM. Bien que cette dynamique soit présente pour les hommes RIM, elle n'a pas la même prépondérance que chez les femmes.

Au-delà de ces premiers éléments de réponse, ce travail d'analyse nous permet de soulever des problèmes qui méritent, selon nous, une attention soutenue si l'on veut effectivement contribuer à l'accroissement des connaissances empiriques dans ce domaine. Le problème central est celui de la nature de la différence. Qu'est-ce que la différence? Où la cherche-t-on ? À quoi la reconnaît-on ? Questions en apparence banales, mais qui soulèvent des difficultés méthodologiques et théoriques importantes.

L'existence de la différence est antérieure à l'intervention pénale. Les rapports sociaux sont des rapports sexués. Les rapports entre les hommes et les femmes dans la société et la place qu'occupe la distinction sur la base du sexe traversent tous les champs du social. À ce titre, le système pénal n'est pas unique. La prémisse de nos analyses est donc que le contrôle social est sexué, qu'il contribue à définir ce qu'est le genre, c'est-à-dire l'identité sociale associée à chacun des sexes.

Il en découle que les diverses formes du contrôle social seraient aussi fort probablement marquées par cette problématique de la différenciation sexuelle. Cette dernière se manifesterait sous des formes spécialisées de marginalité et d'intervention sociale. On aurait ainsi des filières de prédilection, sans pourtant qu'elles soient exclusives, pour les femmes et les hommes. La marginalité des hommes serait de façon prépondérante traitée comme délinquante et orientée vers le système pénal ; la marginalité des femmes serait plutôt traitée comme relevant de la folie ou d'un dysfonctionnement et orientée vers la filière médicopsychiatrique. Ceci permettrait d'expliquer, du moins en partie, la très forte disproportion entre femmes et hommes dans la prise en charge pénale, tout en évitant l'écueil dangereux des explications naturalistes. 
D'un point de vue criminologique, il ne suffit pourtant pas de constater cette disparité entre femmes et hommes dans le volume des contacts pénaux. Il est aussi nécessaire d'étudier les formes de prise en charge en fonction du sexe des justiciables. Ces travaux ne sont pas sans poser des difficultés. D'abord, sur le plan théorique, une approche qui aborderait cette question sous l'angle des privilèges ou de la nonéquité nous mènerait dans un cul-de-sac. L'étude de la différence et de sa traduction dans des profils pénaux particuliers n'implique pas que l'on hiérarchise ces derniers, mais plutôt que l'on mette l'accent sur une plus grande compréhension du fonctionnement du système pénal. De plus, sur le plan méthodologique, ces études se butent, on l'aura constaté dans les pages précédentes, à la difficulté de traiter des phénomènes dont la taille est objectivement très différente. Ceci ne constitue pourtant pas, selon nous, une raison suffisante pour abandonner les analyses empiriques; celles-ci exigent tout simplement encore plus de minutie et le développement de stratégies méthodologiques adaptées.

C'est dans cette perspective que nous avons présenté ici une première analyse du phénomène des réincarcérations multiples des justiciables femmes et hommes. Comment se profile, dans ce cas particulier, la différence ? L'hypothèse qui se dégage de cette exploration exige une réponse à double volet. D'une part, le rapport numérique entre les femmes et les hommes RIM est marqué par une distance encore plus grande que pour l'incarcération en général. C'est donc dire que les cas d'intervention pénale « intense » sont le lot quasi exclusif des hommes. Par contre, une fois inscrites dans cette logique, les femmes semblent subir un traitement plus sévère. Cette tendance se signalerait dans la banalité des délits qui entrânent les mesures d'incarcération plus sévères. En d'autres termes, les femmes correspondent peu à l'image des délinquantes et leur marginalité serait définie et prise en charge à l'intérieur d'une autre logique. Mais une fois définies dans un contexte de délinquance, les transgressions semblent être considérées comme plus «choquantes" et entraînent des mesures plus punitives. Ces résultats signalent selon nous la complexité du traitement sexué des justiciables et de la place particulière et spécifique qu'occupe le pénal dans la reproduction des rapports de sexe. 


\section{BIBLIOGRAPHIE}

COUSINEAU, M.-M., LABERGE, D. et THÉORET, B. (1986), Prison et prisonniers : une analyse de la détention provinciale durant la dernière décennie, Montréal, Cahiers du GRAPPP, $\mathrm{n}^{\circ} 1$.

HAMELIN, M. (1985), Les Quêbécois et Québécoises pénalisés, Montréal, Cahiers de l'École de criminologie, $\mathrm{n}^{\circ} 18$.

HAMELIN, M. (1989), Femmes et prisons, Montréal, éditions du Méridien.

IMBLEAU, M. (1988), Profession: détenue. Analyse de la population des femmes réincarcérées dans les prisons du Québec, Montréal, Cahiers du GRAPPP, $\mathrm{n}^{\circ} 6$.

LABERGE, D. (1990), «Women's Criminality, Criminal Women, Criminalized Women ? Questions in and for a Feminist Perspective », Journal of Human Justice, vol. $2, \mathrm{n}^{\circ} 2$, pp. 37-56.

LANDREVILLE, P. (1982), Le Critère de récidive dans l'évaluation des mesures pénales, Montréal, Cahier de l'École de criminologie, $\mathrm{n}^{\circ} 10$.

LANDREVILLE, P., BLANKEVOORT, V. et PIRES, A., Les Cô̂ts sociaux du système pémal, Montréal, Cahier de l'École de criminologie.

MATHIEU, N.-C. (1989), « Identité sexuelie/sexuée/de sexe ? Trois modes de conceptualisation du rapport entre sexe et genre $»$, in A.-M.. Daume-Richard, M.-C. Hurtig et M.-F. Pichevin (ed.), Catégorisation de sexe et constructions scientifiques, Aix-en-Provence, « Petite collection CEFUP », pp. 109-158.

PARENT, C. (1986), «La protection chevaleresque ou les représentations masculines du traitement des femmes au niveau de la justice pénale », Déviance et sociétê, vol. $X, n^{\circ} 2$, pp. 147-175.

ROY, Sh. (1990), Le Genre comme fondement de la différenciation des formes de contrôle social: l'exemple de l'incarcération, thèse de doctorat, département de sociologie, UQAM. 